\title{
CONTEÚDO DOS CRIADOUROS LARVAIS E COMPORTAMENTO DE ADULTOS DE TOXORHYNCHITES (LYNCHIELLA) HAEMORRHOIDALIS HAEMORRHOIDALIS (FABRICIUS) (DIPTERA, CULICIDAE) NUMA FLORESTA DE TERRA-FIRME DA AMAZÔNIA CENTRAL
}

\author{
Rosa Sá Gomes Hutchings ${ }^{1}$
}

\begin{abstract}
LARVAL BREEDING SITE CONTENTS AND ADULT BEHAVIOR OF TOXORHYNCHITES (LYNCHIELLA) HAEMORRHOIDALIS HAEMORRHOIDALIS (FABRICIUS) (DIPTERA, CULICIDAE) IN AN UPLANI FOREST OF THE CENTRAL. AMAZON. The natural breeding sites of Toxorhynchites (Lynchiella) haemorrhoidalis haemorhoidalis (Fahricius. 1794), in two study areas, were sampled monthly, during a period of one year, in an upland "terra-firme" forest of the Central Amazon. These natural breeding sites. consisting of water filled palm bracts on the ground, contained invertebrates and vertebrates along with palm inflorescences, leaves and twigs. The inhabitants of the non-submersed area of the bracts include Diplopoda, Acarina, Araneae, Pseudoscorpiones, Isopoda, Blattodea, Coleoptera (Carabidae, Curculionidae, Scolytidac, Staphilinidae), Collembola, Dermaptera, Diptera (Cecidomyidae, Drosophilidae, Mycetophilidae, Tipulidae), Hemiptera, Hymenoptera and Trichoptera. The suhmersed areas of the bracts were inhabited by Oligochaeta, Coleoptera (Dysticidae. Helodidae, Histeridae, Hydrophilidae, Limnebiidae), Diptera (Ceratopogonidae, Chironomidae, Culicidae, Psychodidae, Stratiomyidae, Syrphidae), Odonata, along with immature Dendrohatidae e Hylidae. The ovipositing, resting and feeding behaviors of $T$. h. haemorrhoidalis adults are described.
\end{abstract}

KEY WORDS. Toxorhynchites haemorrhoidalis, breeding sites, behavior, Amazon

Os estudos sobre Toxorhynchites Theobald, 1901 baseiam-se principalmente nas larvas, as quais são predadoras de imaturos de outros mosquitos e de pequenos artrópodes. Considerando que os adultos não possuem hábitos hematófagos, eles têm o potencial de serem agentes de controle biológico de outros culicídeos (StefFan 1975; StefFan \& Evenhuis 1981; Hutchings 1991). Das quatro espécies de Toxorhynchites registradas para a região amazônica brasileira (CERQUEIRA 1961), Toxorhynchites (Lynchiella) haemorrhoidalis haemorrhoidalis (Fabricius, 1794) ocorre em Manaus, Amazonas onde utiliza brácteas de palmeiras como criadouros naturais juntamente com outros invertebrados e vertebrados, e ainda vários criadouros artificiais (HuTCHINGS 1990). Os comportamentos de predação, canibalismo e emergência de T. h. haemorrhoidalis no laboratório junto com dados sobre o desenvolvimento e a criação desta espécie no laboratório foram apresentados por HuTCHINGs (1993). A disponibilidade e durabilidade das brácteas de palmeiras e a dinâmica populacional das larvas desta espécie nestes criadouros naturais foram descritos por HUTCHINGS (1994).

1) Coordenadoria de Pesquisa em Entomologia. Instituto Nacional de Pesquisas da Amazônia, Caixa Postal 478, 69011-970 Manaus, Amazonas, Brasil. 
O presente trabalho visa complementar as informações disponíveis sobre esta espécie com o objetivo de identificar a fauna existente nos criadouros utilizados pelo $T$. h. haemorrhoidalis e de descrever o seu comportamento de oviposição, repouso e alimentação.

\section{MATERIAL E MÉTODOS}

As coletas nos criadouros naturais e as observações de comportamento dos adultos foram efetuadas na Reserva Florestal Adolpho Ducke, localizada a $26 \mathrm{Km}$ ao norte do município de Manaus $\left(02^{\circ} 55^{\prime} \mathrm{S}, 59^{\circ} 59^{\prime} \mathrm{W}\right)$ pela Rodovia Torquato Tapajós (AM-010) (ARAujo 1967). A época da seca nesta região, ocorre entre junho e novembro e a época de chuva entre dezembro e maio. A precipitação pluvial média anual é de $2105 \mathrm{~mm}$, com $75 \%$ ocorrendo durante a estação chuvosa (RIBEIRO \& AdIS 1984). Dados obtidos do Boletim Meteorológico do INPA Estação Meteorológica da Reserva Ducke (1986, mimeografado) revelam que a precipitação mínima ocorreu no mês de agosto $(41,5 \mathrm{~mm})$ e a máxima no mês de novembro $(445,8 \mathrm{~mm})$. A umidade relativa do ar foi quase constante durante o ano, com a mínima no mês de agosto $(73,8 \%)$ e a máxima no mês de fevereiro $(86,8 \%)$. A temperatura média mensal manteve-se relativamente constante durante todo o ano $\left(25,3^{\circ}, 27,5^{\circ} \mathrm{C}\right)$.

Foram delimitadas duas áreas de estudo de $50 \times 50 \mathrm{~m}$ onde as palmeiras foram numeradas, mapeadas e identificadas (ver detalhes em HuTCHINGs 1994). A área 1 (denominada de "bosque") é constituída de árvores de grande porte, algumas cultivadas, outras nativas e palmeiras. Esta área é bosqueada regularmente, não tendo sub-bosque. A área 2 (denominada de "baixio") é atravessada por um igarapé formando assim áreas alagadiças contendo árvores de grande porte e palmeiras de várias espécies.

Os criadouros, brácteas de palmeiras que ao cair no chão retém água pluvial, foram coletados mensalmente no período de janeiro a dezembro de 1986. No primeiro mês, foi escolhida uma bráctea com material para se fazer a coleta; no mês seguinte, coletou-se na mesma bráctea do mês anterior e em uma segunda; no terceiro mês, repetiram-se a primeira e segunda brácteas, e em uma terceira, prosseguindo assim até completar 12 meses.

No laboratório, foi feita a triagem do material coletado nas brácteas separando larvas de Toxorhynchites, larvas de outros culicídeos, outros invertebrados, vertebrados e o material orgânico existente. Os registros das larvas de Toxorhynchites coletadas são apresentados e discutidos em HuTCHINGs (1994). Os outros culicídeos foram separados em copos e colocados em gaiolas com água do próprio criadouro até a emergência do adulto para serem montados e identificados. Os demais invertebrados presentes nas coletas foram triados e identificados, quando possível, até nível de família.

Observações sobre oviposição, repouso e alimentação foram feitas nos dias em que as brácteas foram amostradas nas duas áreas de estudo. A atividade dos adultos era acompanhanda, das 6:00 as 18:00 horas, mensalmente durante um dia em cada área. 


\section{RESULTADOS E DISCUSSÃO}

\section{CONTEÚDO DAS BRÁCTEAS}

O conteúdo das brácteas coletadas incluiu organismos pertencentes a três filos diferentes: Annelida, Arthropoda e Chordata (Tab. I). A matéria orgânica coletada, junto com estes organismos, consistia em inflorescências das palmeiras, folhas e pedaços de gravetos.

Os anelídeos, representados pelos Oligochaeta, estiveram presentes nas duas áreas de estudo, porém somente ocorreram durante um mês em cada local: abril e fevereiro, nas áreas 1 (bosque) e 2 (baixio), respectivamente.

Entre os artrópodes ocorreram Diplopoda, Acarina, Araneae, Pseudoscorpiones, Isopoda, Blattodea, Coleoptera (Carabidae, Curculionidae, Scolytidae, Staphilinidae), Collembola, Dermaptera, Diptera (Cecidomyidae, Drosophilidae, Mycetophilidae, Tipulidae), Hemiptera, Hymenoptera e Trichoptera hahitando as partes não submersas das brácteas. Entre os coleópteros, que estiveram presentes na água das brácteas, as famílias Histeridae e Limnebiidae restringiram-se apenas à área 2 (baixio), enquanto as famílias Dysticidae, Helodidae e Hydrophilidae ocorreram nas duas áreas. Os dípteros que ocorreram na água das brácteas, nas duas áreas, incluíram indivíduos das famílias Ceratopogonidae, Chironomidae, Culicidae, Psychodidae, Stratiomyidae e Syrphidae porém com uma maior diversidade na área 2 (baixio). A ordem Odonata foi encontrada em julho na área 2 (baixio), enquanto que ná área 1 (bosque) ocorreu durante vários meses do ano.

Os membros do filo Chordata, representados pelos anuros das famílias Dendrobatidae e Hylidae, tiveram padrões de presença sazonais, ocorrendo somente na área 1 (bosque).

LOPES et al. (1983, 1985) assinalaram cinco gêneros de Culicidae em criadouros artificiais, juntamente com T. h. haemorrhoidalis. Em criadouros artificiais colocados em diferentes alturas (nível do chão até onze metros) as larvas de $T$. h. haemorrhoidalis só foram registradas entre um a seis metros, estando ausentes ao nível do chão e de sete a onze metros. Os cinco gêneros de culicídeos registrados por LOPES et al. $(1983,1985)$ também ocorreram na presente pesquisa de criadouros ao nível do solo.

LOUNIBOS et al. (1987a, 1987b) assinalaram para a Venezuela a presença da larva de $T$. haemorrhoidalis associada com larvas de Diptera (Culicidae, Syrphidae, Psychodidae, Ceratopogonidae, Tipulidae, Stratiomyidae, Thaumaleidae, Chironomidae, Ephydridae, Tabanidae), Coleoptera (Helodidae, Hydrophilidae), Odonata (Coenagrionidae), Platyhelminthes, Oligochaeta e com Acarina, sem especificar o estágio de desenvolvimento dos mesmos e concluíram que a predação por larvas de $T$. haemorrhoidalis reduziu significativamente a abundância de Culicidae, Ceratopogonidae, Psychodidae, Thaumaleidae e Chironomidae. Comparando os presentes resultados com Lounibos et al. (1987a, 1987b), notou-se que a diversidade, a nível de família, foi bastante similar com exceção da presença de nove famílias de Coleoptera, contra duas na Venezuela, e de duas famílias diferentes de Odonata. É importante ressaltar a ausência de Anura nas coletas feitas por LouniBOS et al. (1987b) podendo ser devida à diferença nos 
Tab. I. Ocorrência mensal de invertebrados e vertebrados nas coletas em brácteas de palmeiras, criadouros naturais de Toxorhynchites h. haemorrhoidalis, nas áreas de estudo da Reserva Ducke durante 1986 na Amazônia Central.

\begin{tabular}{|c|c|c|c|c|c|c|c|c|c|c|c|c|c|}
\hline Grupo & Familia & Gênero & Fase & Jan & n Fev & Mar & Abr & Mai Jun & Jul Ago* & Set* & Out * & Nov & Dez* \\
\hline Oligochaeta & ? & & $\mathrm{Ad}$ & & $x$ & & 0 & & & & & & \\
\hline Diplopoda & $?$ & & $A d$ & & & & & & & 0 & 0 & & \\
\hline Acarina & Gamasidae & & $\mathrm{Ad}$ & & & & xo & $x$ & $x$ & & & & \\
\hline Araneae & $?$ & & Ad & & & & 0 & & 0 & & 0 & & \\
\hline Pseudoscorpiones & Alpiidae & & $\mathrm{Ad}$ & & & & & $x$ & & & & & \\
\hline Isopoda & ? & & $\mathrm{Ad}$ & $x$ & & & & & & & & & \\
\hline \multirow[t]{5}{*}{ Blatt odea } & Blattidae & & Ad & & $x$ & & xo & & & & & & \\
\hline & Carabidae & & $\mathrm{Ad} / \mathrm{Im}$ & & & $x$ & & & & & & & \\
\hline & Curculionidae & & Im & & $x$ & $x$ & $x$ & & 0 & 0 & & & \\
\hline & Dysticidae & & Ad & 0 & xo & $x$ & xO & xo & xo & $\mathrm{O}$ & 0 & & 0 \\
\hline & Helodidae & & Im & 0 & & xo & xo & $0 \times 0$ & xo & $\mathrm{O}$ & 0 & & 0 \\
\hline \multirow[t]{5}{*}{ Coleoptera } & Histeridae & & Ad & & $x$ & $x$ & $x$ & & & & & & \\
\hline & Limnebridae & & Ad & & & & & & $x$ & & & & \\
\hline & Hydrophilidae & & Ad & & $x$ & $x$ & xO & 0 & xo & 0 & o & & \\
\hline & Scolytidae & & Ad & & & & xo & & & 0 & & & \\
\hline & Staphilinidae & & $\mathrm{Ad}$ & 0 & & & $x$ & & & & & & \\
\hline Collembola & Poduridae & & $\mathrm{Ad}$ & 0 & & & & & & & 0 & & 0 \\
\hline \multirow[t]{8}{*}{ Dermaptera } & Forficulidae & & $\mathrm{Ad}$ & & & & & $x$ & & $\mathrm{O}$ & 0 & & o \\
\hline & $?$ & & $A d$ & & $x$ & & $x$ & & & & & & \\
\hline & Cecidomyidae & & Ad & & & & & & & 0 & 0 & & \\
\hline & Ceratopogonidae & Artrichopogon & Im & & & $x$ & $x$ & & & & & & \\
\hline & & Forcipomyia & $\mathrm{Im}$ & & & & $\mathrm{x}$ & & & & & & \\
\hline & Chironomidae & & $\mathrm{Im}$ & xo & $x$ & xo & xo & $0 \quad 0$ & xo & 0 & & & 0 \\
\hline & & Anopheles & $\mathrm{Im}$ & & & xo & xo & & 0 & & & & \\
\hline & & Culex & $\mathrm{Im}$ & & xo & xo & xo & $x$ & xo & & & 0 & 0 \\
\hline \multirow[t]{10}{*}{ Diptera } & Culicidae & Limatus & Im & & & & $x$ & & $x$ & & & & o \\
\hline & & Toxorhynchites & $\mathrm{Im}$ & xo & $\times 0$ & xo & xo & xo & xo & $x$ & & & \\
\hline & & Trichoprosopon & Im & & xo & & & & 0 & & & & \\
\hline & & Wyeomyia & Im & & & & & & o & & & & \\
\hline & Drosophilidae & & $\mathrm{Ad}$ & & & & $x$ & & & & & & \\
\hline & Mycetophilidae & & $\mathrm{Ad}$ & & & & $x$ & & & & & & \\
\hline & Psychodidae & & Im & $x$ & $x$ & $x$ & $x$ & $x$ & $x$ & & & & \\
\hline & Stratiomyidae & & Im & & & $x$ & & $x$ & & & & & \\
\hline & Syrphidae & & $\mathrm{Im}$ & & & & & & & 0 & & & \\
\hline & Tipulidae & & Ad & & & & $x$ & $\mathrm{x}$ & & & & & \\
\hline Hemiptera & $?$ & & $\mathrm{Ad}$ & 0 & $x$ & & $x$ & $x$ & & & & & \\
\hline Hymenoptera & $?$ & & $A d$ & o & & & & o & & 0 & 0 & & o \\
\hline \multirow[t]{2}{*}{ Odonata } & Aeshnidae & & Im & 0 & & & 0 & 0 & xo & 0 & 0 & & o \\
\hline & Libellulidae & & Im & 0 & o & & & 0 & & & o & & o \\
\hline Trichoptera & Hydropsychidae & & Ad & & & & & & 0 & & & & \\
\hline \multirow[t]{2}{*}{ Anura } & Dendrobatidae & Dendrobates & $\mathrm{Im}$ & 0 & 0 & & & & & & o & & o \\
\hline & Hylidae & Osteocephalus & $\mathrm{Im}$ & 0 & & 0 & 0 & & & & 0 & & 0 \\
\hline
\end{tabular}

(?) Não identificado, (O) presente na Área 1, (X) presente na Área 2, ( ${ }^{*}$ ) sem criadouro disponível com água na Área 2, (Im) imaturo, (Ad) adulto.

Revta bras. Zool. 12 (2): 313 - 319, 1995 
criadouros amostrados (bráctea de Heliconia, duas espécies de Aechmea e internódio de Bambusa). Considerando-se que as informações sobre o contelído de brácteas é qualitativa, e não quantitativa, não foi possível testar correlações entre a presença de presas e predadores nos mesmos criadouros.

A baixa precipitação pluvial do mês de agosto pode ter influenciado sobre a baixa ocorrência de invertebrados nos criadouros nas duas áreas; durante este mês só se tem registro de culicídeos.

\section{COMPORTAMENTO DOS ADULTOS}

As observações sobre o comportamento da fềmea de $T$. h. haemorrhoidalis na desova, mostraram que o horário de postura é vespertino, no período das 14:00 às 18:00 horas. A fêmea se aproxima da bráctea com água, sobrevoa toda sua abertura e inicia o vôo característico da desova com movimentos de subidas e descidas sucessivas, sem tocar na superfície da água, e a cada descida ejeta um ovo. Fêmeas coletadas na natureza desovaram em gaiolas no laboratório, mostrando o mesmo comportamento da oviposição.

No levantamento preliminar de criadouros potenciais, efetuado na Reserva Ducke, encontrou-se uma preferência pelos criadouros localizados ao nível do solo; em apenas dois criadouros localizados acima do nível do solo, dos mais de 100 observados, coletaram-se larvas de Toxorhynchites. As brácteas onde havia decomposição de matéria orgânica, com forte odor de putrefação, e de cor escura, eram as preferidas para ovipositar, mesmo quando ao lado havia outra bráctea sem estas características.

Não foram observadas duas fêmeas ovipositando simultaneamente num mesmo criadouro. Se uma fềmea está ovipositando e chega uma segunda, a primeira fêmea interrompe a oviposição e fica pousada próxima ao criadouro, retornando na maioria das vezes, para ovipositar quando da saída da segunda. Este comportamento não foi anteriormente registrado para esta espécie, nem para outra espécie do gênero. Observou-se que as fêmeas interrompem a oviposição nos períodos de chuva e, na maioria das vezes, o excesso da água pode jogar os ovos para fora do criadouro. Esta interferência da chuva também foi registrada por Lounibos et al. (1987b), onde os ovos de T. haemorrhoidalis colocados nas brácteas de Heliconia sp. foram jogados para fora das mesmas durante as chuvas pelo excesso de água. Não foi encontrado registro na literatura descrevendo o comportamento de oviposição de $T$. h. haemorrhoidalis, porém as descrições existentes sobre a oviposição em outras espécies do genêro indicam um comportamento similar entre elas (BONNET \& HU 1951; FOCKS \& BOSTON 1979; STEFFAN et al. 1980; TRPIS 1981; STEFFAN \& EvENHUIS 1981).

As fêmeas observadas ovipositando, nos criadouros na natureza (brácteas), depositaram um mínimo de um e um máximo de doze ovos por postura com uma

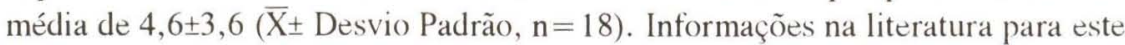
gênero, sobre o número de ovos são escassas. Dados publicados para a espécie $T$. brevipalpis indicam que cada fềmea pode depositar quinze ovos num período relativamente pequeno (TRPIS \& GERBER 1973 apud MACHADO-ALLISON 1980).

Ao pousar numa folha, o macho e a fêmea apoiam-se nas pernas anteriores 
e médias. As posteriores ficam suspensas com movimentos leves para cima e para baixo, alternando a perna esquerda e direita. Ao voar, o macho e a fềmea podem produzir um "zumbido" característico.

Tanto a fêmea como o macho de T. h. haemorrhoidalis alimentam-se de néctar de flores na natureza. Em cativeiro aceitam substâncias açucaradas e/ou frutas como mamão, maçã e melancia as quais foram oferecidas durante as criações. Na floresta observou-se a espécie alimentando-se nas flores de uma Violaceae, Rinoria flavescens (Albl.) O. Kuntze, e em uma Rubiacede, Borreria verticiliata (G.F.W.Mey) (nome vulgar - vassourinha de botão). MACHADOALLISON (1982) confirma a alimentação à base de néctar e outras substâncias açucaradas pelos adultos de Toxorhynchites. Existem poucos registros de observações de campo sobre alimentação no gênero e não foi encontrada nenhuma observação para $T$. $h$. haemorrhoidalis. A descoberta de $B$. verticiliata, uma erva comum de fácil obtenção, como fonte de alimento natural de $T$. h. haemorrhoidalis poderá ajudar na criação desta espécie em cativeiro.

AGRADECIMENTOS. Agradeço ao Dr. José A.S. Nunes de Mello pelo incentivo para o desenvolvimento deste trabalho; a Nair O. Aguiar pela identificação dos Pseudoscorpiones e Coleoptera: a Eloy Castellon pela identificação dos Ceratopogonidae: a Hugo Mesquita pela identificação das Odonata; e a Jean-Mare Hero pela identificação dos Anura.

\section{REFERÊNCIAS BIBLIOGRÁFICAS}

ARAujo, V.C. DE. 1967. A Reserva Florestal Ducke (Manaus): Características e principais elementos florísticos e faunísticos protegidos. Atas do Simpósio sobre a Biota Amazônica (Conservação da Natureza e Recursos Naturais) 7: 57-68.

BONNET, D.D. \& S.M.K. Hu. 1951. The introduction of Toxorhynchites brevipalpis Theobald into the Territory of Hawaii. Proc. Hawaiian ent. Soc. 14 (2): 237-242.

Cerqueira, N.L. 1961. Distribuição geográfica dos mosquitos da Amazônia (Diptera, Culicinae). Revta bras. Ent. 10: 111-168.

Focks, D.A. \& M.D. Boston. 1979. A quantified mass-rearing technique for Toxorhynchites rutilus rutilus (Coquillett). Mosq. News 39 (2): 616-619.

Hutchings, R.S.G. 1990. Observações Biológicas de Toxorhynchites (Lynchiella) haemorrhoidalis haemorrhoidalis (Fabricius, 1794) (Diptera: Culicidae) numa Floresta de Terra-firme da Amazônia Central e no Laboratório. Dissertação de Mestrado, não publicada, Instituto Nacional de Pesquisas da Amazônia, Fundação Universidade do Amazonas, Manaus, 89p. 1991. Possibilidades do uso de espécies do gênero Toxorhynchites Theobald, 1901 (Diptera: Culicidae) como controladores biológicos de Culicidae na Amazônia, p. 383-385. In: A.L. Val; R. Figliuolo \& E. Feldberg (eds). Bases Científicas para Estratégias de Preservação e Desenvolvimento da Amazônia: Fatos e Perspectivas. Vol. I. Manaus, INPA, Imprensa Universitária Universidade do Amazonas, 440p. 
1993. Desenvolvimento, criação e comportamento de Toxorhynchites (Lynchiella) haemorrhoidalis haemorrhoidalis (Fabricius, 1794) (Diptera: Culicidae) no laboratório. Acta Amazônica 23 (1): 37-47.

1994. Breeding sites and their exploitation by Toxorhynchites (Lynchiella) haemorrhoidalis haemorrhoidalis (Diptera: Culicidae) in an upland forest of the Central Amazon. J. Med. Ent. 31 (2): 186-191.

LOPES, J.; R.J. ARIAS \& J.D. CHARLWOOD. 1983. Evidências preliminares de estratificação vertical de postura de ovos por alguns Culicidae (Diptera) em floresta no Municipio de Manaus-Amazonas. Acta Amazônica 13 (2): 431-439.

1985. Estudo ecológico de Culicidae (Diptera) silvestres criando em pequenos recipientes de água em mata e em capoeira no município de Manaus - AM. Ciência e Cultura 37 (8): 1299-1311.

Lounibos, L.P.; J.H. Frank; C.E. Machado-Allison; J.C. Navarro \& P. OCANTO. 1987a. Seasonality, abundance and invertebrate associates of Leptagrion siqueirai Santos in Aechmea bromeliads in Venezuelan rain forest (Zygoptera: Coenagrionidae). Odonatologica 16 (2): 193-199.

Lounibos, L.P.; J.H. FranK; C.E. Machado-Allison; P. Ocanto \& J.C. NAVARRO. 1987b. Survival, development and predatory effects of mosquito larvae in Venezuelan phytotelmata. J. Trop. Ecol. 3 (3): 221-242.

Machado-Allison, C.E. 1980. Ecologia de los mosquitos (Culicidae). I. Huevos y oviposición. Acta biol. venez. 10 (3): 303-371.

- 1982. Ecologia de los mosquitos (Culicidae). III. Adultos. Acta biol. venez. 11 (3): 133-237.

RIBEIRO, M.N.G. \& J. ADIS. 1984. Local rainfall variability - A potential bias for bio-ecological studies in the Central Amazon. Acta Amazonica 14 (1-2): 159-174.

STEFFAN, W.A. 1975. Systematics and biological control potential of Toxorhynchites (Diptera: Culicidae). Mosq. Syst. 7 (1): 59-67.

Steffan, W.A. \& N.L. Evenhuis. 1981. Biology of Toxorhynchites. Ann. Rev. Ent. 26: 159-181.

STEFFAN, W.A.; R.D. STOAKS \& N.L. Evenhuis. 1980. Biological observations of Toxorhynchites amboinensis (Diptera: Culicidae) in the laboratory. J. Med. Ent. 17 (6): 515-518.

TRPIS, M. 1981. Survivorship and age specific fertility of Toxorhynchites brevipalpis females (Diptera: Culicidae). J. Med. Ent. 18 (6): 481-486. 